\title{
On a Group of Transformations connected with the 27 Lines of the Non-Singular Cubic Surface.
}

\author{
By Dr J. F. Tixto.
}

(Read 8th June 1917. Received 12th July 1917.)

\section{Introductory.}

In the paper, "Transformations founded on the Twisted Cubic and its Chord System," a series of space transformations was described, each of which bad the property of transforming the chord system of one cubic into the chord system of the other. In the present paper it is shown that by the aid of a non-singular cubic surface the transformations of orders $1,2,3,4,5$ may be derived directly without the intervention of a space transformation.

It will be found that these transformations form a group which is intimately associated with the 27 lines of the cubic.

\section{Derivation of the Transformations.}

Consider a cubic surface without conical points or other singularities, $F_{\mathbf{3}}$.

Let there be taken on it two twisted cubics $\Sigma_{3}, \Sigma_{3}^{\prime}$ intersecting in $r$ points where $r$ may have any of the 5 values $1,2,3,4,5$. No other values of $r$ are possible.

Any chord $\rho$ of $\Sigma_{3}$ meets $F_{3}$ in a single point $P$, and through $P$ we can draw a unique chord $\rho^{\prime}$ of $\Sigma_{3}^{\prime}$. $\quad \rho$ and $\rho^{\prime}$ thus correspond, and it is clear that the correspondence is $(1,1)$. Thus by means of the points of $F_{3}$ we can establish a $(1,1)$ relationship between the chord systems of $\Sigma_{3}$ and $\Sigma_{3}^{\prime}$.

Let a point $Q$ describe an arbitrary line. The chord system of $\Sigma_{3}$ through it constitutes a ruled quartic which has $\Sigma_{3}$ as double curve. This surface intersects $F_{3}$ in a rational sextic without actual double points, which meets $\Sigma_{3}$ in 10 points.

$\Sigma_{3}^{\prime}$ meets the quartic in 12 points, of which $2 r$ lie on $\Sigma_{3}$. Hence there remain $12-2 r$ points of intersection, and these must lie on the rational sextic. Consequently, the chords of $\Sigma_{3}{ }^{\prime}$ through 
the sextic generate a ruled surface of order $4 \times 6-2(12-2 r)$, i.e. $4 r$.

The ruled quartic through $\Sigma_{z}$ therefore transforms into a ruled surface of order $4 r$, and so the transformation between the chord systems is of order $r$. We thus see that transformations of orders 1 to 5 are possible.

3. F-chords of a transformation.

The number and order of the $F$-chords of a transformation of order $r$ are identical respectively with the number and order of the $F$-points of a plane Cremona transformation of order $r$.

The correspondent of a chord of $\searrow_{3}$ may not be a unique chord of $\Sigma_{3}^{\prime}$, but a surface of chords. This is only possible if the chord of $\Sigma_{3}$ be a line on $F_{33}$. Further, the chord of $\Sigma_{3}$, which is a $F$-chord, cannot also be a chord of $\Sigma_{3}^{\prime}$, and hence it either does not intersect $\Sigma_{3}^{\prime}$ or it intersects it in 1 point only. There are thus two types of $F$-chord possible, and two only, viz. $(a)$ a chord of $\Sigma_{3}$ which does not intersect $\Sigma_{3}^{\prime}$, and whose corresponding surface is a ruled quartic. This chord corresponds to a $F$-point of order 2 in a Cremona transformation. (b) A chord of $\Sigma_{3}$ which intersects $\Sigma_{a}^{\prime}$ in 1 point, and whose corresponding surface is a conicoid. This chord corresponds to a $F$-point of order 1 .

4. The F-systems of the various transformations.

(i) The quintic transformation : $\Sigma_{3}, \Sigma_{3}$ intersect in 5 points.

There must exist 6 chords of $\Sigma_{3}$, which are at the same time lines of $F_{3}$ which do not intersect $\Sigma_{3}$, and there are 6 similar chords of $\Sigma_{3}^{\prime}$ These 12 lines constitute a double six. For, corresponding to a chord-line of $\Sigma_{3}$ we obtain a quartic which contains 5 of the chord-lines of $\Sigma_{3}{ }^{\prime}$. Hence any chord-line of $\Sigma_{3}$ intersects 5 of the chord-lines of $\Sigma_{3}^{\prime}$.

The remaining 15 lines on the surface must each meet $\Sigma_{3}, \Sigma_{3}^{\prime}$ in 1 point. It is easy to show that any one of them meets 6 of the remaining 14.

(ii) The quartic transformation: $\Sigma_{3}, \Sigma_{3}{ }^{\prime}$ intersect in 4 points.

There must exist 3 chord-lines of $\Sigma_{3}$ which do intersect $\Sigma_{3}{ }^{\prime}$, and vice-versa; and 3 which intersect $\Sigma_{3}^{\prime}$ in 1 point, and vice-versa.

Let these lines be denoted by

$$
\left\{\begin{array}{l}
{ }_{2} L_{1},{ }_{2} L_{2},{ }_{2} L_{3},{ }_{1} L_{1},{ }_{1} L_{2},{ }_{1} L_{3}, \\
{ }_{2} L_{1}^{\prime},{ }_{2} L_{2}^{\prime},{ }_{2} L_{3}^{\prime},{ }_{1} L_{1}^{\prime},{ }_{1} L_{2}^{\prime},{ }_{1} L_{3}^{\prime},
\end{array}\right.
$$

where the prefixes indicate order. 
Corresponding to ${ }_{2} L_{k}(s=1,2,3)$ we must have a quartic which contains ${ }_{2} L_{1}^{\prime},{ }_{2} L_{2}^{\prime},{ }_{2} L_{3}^{\prime}$ and two of ${ }_{1} L_{n}^{\prime}(s=1,2,3)$, say those with a different suftix. Corresponding to , $L,(s=1,2,3)$ we must have a conicoid containing two of the lines ${ }_{2} L !$, , say the two with different suffixes.

Hence we infer that ${ }_{2} L_{4}$ meets ${ }_{2} L_{1}{ }_{1},{ }_{2} L_{2}^{\prime},{ }_{2} L_{3}^{\prime}$ and two of ${ }_{1} L_{4}^{\prime}$, and that $L_{3}$ meets two of ${ }_{2} L_{s}$.

To discover the relation of the remaining 15 lines to the above 12, we form the double six

$$
\left\{\begin{array}{l}
{ }_{2} L_{1},{ }_{2} L_{2},{ }_{2} L_{3}, M_{23}, M_{31}, M_{12}, \\
M_{23}, M_{31}^{\prime}, M_{12}^{\prime}, \quad{ }_{2} L_{1}^{\prime},{ }_{2} L_{2}^{\prime},{ }_{2} L_{3}^{\prime},
\end{array}\right.
$$

where $M_{23}$ are 6 additional lines to be determined.

The 6 lines which are the third lines of the planes determined by $\left|M_{31},{ }_{2} L_{3}^{\prime}\right|,\left|M_{12},{ }_{2} L_{1}^{\prime}\right|$, etc., are identical with ${ }_{1} L_{1},{ }_{1} L_{2}$, etc., and hence $M_{31}$ is determined as the third line of the plane $\left|{ }_{1} L_{1},{ }_{2} L_{3}\right|$, etc.

The lines $M_{31}$, etc., meet $\Sigma_{3}$ in 1 point, but do not intersect $\aleph_{3}^{\prime}$, and vice-versa for the three $M_{31}$.

The remaining 9 lines are determined by the planes

$$
\left|{ }_{2} L_{1},{ }_{2} L_{1}^{\prime}\right|,{ }_{2} L_{1},{ }_{2} L_{2}^{\prime} \mid \text {, etc. }
$$

They each meet both $\Sigma_{3}$ and $\Sigma_{3}^{\prime}$ in 1 point. Any one meets 6 of the remaining 8 .

(iii) The cubic transformation: $\Sigma_{3}$ and $\Sigma_{3}$ intersect in 3 points.

There must exist one chord-line of $\Sigma_{3}$, which does not meet $\Sigma_{3}^{\prime}$; and 4 chord-lines which each meet $\Xi_{3}^{\prime}$ in one point. There is a

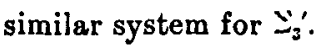

Since any cubic on $F_{3}$ possesses 6 chord-lines, there will be one line of $F_{3}$ which is a chord-line of both $\Sigma_{3}$ and $\Sigma_{3}^{\prime}$.

Let the $F$-system be

$$
\left\{\begin{array}{l}
{ }_{2} L_{1},{ }_{1} L_{1},{ }_{1} L_{2},{ }_{1} L_{3},{ }_{1} L_{4}, \\
{ }_{2} L_{1}^{\prime},{ }_{1} L_{1}^{\prime},{ }_{1} L_{2}^{\prime},{ }_{1} L_{3}^{\prime},{ }_{1} L_{4}^{\prime} .
\end{array}\right.
$$

${ }_{2} L_{1}$ must meet the 5 lines ${ }_{\mathrm{g}} L_{1}{ }_{1},{ }_{1} L_{1}{ }^{\prime}$, etc. ${ }_{1} L_{s}(s=1 \ldots 4)$ must meet ${ }_{2} L_{1}$, and one of the $4{ }_{1} L_{c}$, say the one with the same suffix.

Let $M_{6}$ denote the common chord-line of both cubics. We form the double sixes

(1) $\left\{\begin{array}{l}{ }_{2} L_{1}, M_{5}, M_{1}, M_{2}, M_{3}, M_{4}, \\ M_{6}^{\prime},{ }_{2} L_{1}^{\prime},{ }_{2} L_{1}^{\prime},{ }_{1} L_{2}^{\prime},{ }_{1}^{\prime} L_{3}^{\prime},{ }_{1} L_{4}^{\prime} .\end{array}\right.$
(2) $\left\{\begin{array}{l}{ }_{2} L_{1}^{\prime}, M_{5}^{\prime}, M_{1}, M_{2}^{\prime}, M_{3}^{\prime}, M_{4}^{\prime}, \\ M_{6},{ }_{2} L_{1},{ }_{2} L_{1},{ }_{1} L_{2},{ }_{1} L_{3},{ }_{1} L_{4} .\end{array}\right.$ 
$M_{5}$ and $M_{5}^{\prime}$ are evidently identical. Each is the second transversal of the group ${ }_{1} L_{1},{ }_{1} L_{2},{ }_{2} L_{3},{ }_{1} L_{4}$ (the first being ${ }_{2} L_{1}^{\prime}$ ).

This line $M_{5}$ cannot meet either $\Sigma_{3}$ or $\Sigma_{3}$, and it is the only line of this kind.

The lines $M_{1}(s=1 \ldots 4)$ meet $\Sigma_{3}$ in 1 point, but do not meet $\Sigma_{3}^{\prime}$. Similarly for $M_{:}^{\prime}$.

The lines determined by the planes $\left|{ }_{2} L_{1},{ }_{1} L_{c}^{\prime}\right|(s=1 \ldots 4)$ are identical with $M_{4}$, and the lines determined by $\left|{ }_{2} L_{1}^{\prime},{ }_{1} L_{s}\right|$ are identical with $M_{:}^{\prime}$.

The six lines determined by the planes $\left|M_{s},{ }_{1} L_{s}^{\prime}\right|(s=1 \ldots 4)$ meet $\Sigma_{3}$ and $\Sigma_{3}{ }^{\prime}$ each in one point.

The 4 lines determined by the planes $\left|M_{5},{ }_{1} L_{a}^{\prime}\right|$ are identical with ${ }_{1} L_{s}(s=1 \ldots 4)$.

The line determined by the plane $\left|\boldsymbol{M}_{5}, M_{0}\right|$ is the 27 th line, and it meets $\Sigma_{3}$ and $\Sigma_{3}^{\prime}$ each in one point.

(iv) The quadratic transformation: $\Sigma_{3}$ and $\Sigma_{3}^{\prime}$ intersect in two points.

There are 3 chord-lines of $\check{\beth}_{3}$ which intersect $\Sigma_{3}^{\prime}$ in one point, and vice-versa.

There are also 3 chord-lines of $\Sigma_{3}$ which are at the same time chord-lines of $\Sigma_{3}^{\prime}$.

Let the $F$-system be

$$
\left\{\begin{array}{l}
{ }_{1} L_{1},{ }_{1} L_{2},{ }_{1} L_{3}, \\
{ }_{1} L_{1}^{\prime},{ }_{1} L_{2}^{\prime},{ }_{2} L_{3}^{\prime} .
\end{array}\right.
$$

Any one of ${ }_{1} L$, must meet 2 of ${ }_{1} L_{\mathfrak{s}}^{\prime}$, and conversely.

Construct the double six

$$
\left\{\begin{array}{l}
{ }_{1} L_{1},{ }_{1} L_{2},{ }_{1} L_{3}, M_{4}, M_{5}, M_{6}, \\
{ }_{1} L_{1}^{\prime},{ }_{1} L_{2}^{\prime},{ }_{1} L_{3}^{\prime}, M_{4}^{\prime}, M_{5}^{\prime}, M_{6}^{\prime} .
\end{array}\right.
$$

The lines $M_{3}$ intersect $\Sigma_{3}$ in l point, but not $\Sigma_{3}^{\prime}$, and conversely for the lines $M_{s}^{\prime}$.

The 3 lines determined by the planes $\left|{ }_{1} L_{1},{ }_{1} L_{2}^{\prime}\right|$ do not meet $\Sigma_{3}$ or $\Sigma_{3}^{\prime}$, and they are the only three of this kind.

The 9 lines determined by the planes $\left|{ }_{1} L_{1}, M_{r}^{\prime}\right|$ meet $\Sigma_{3}$ and $\Sigma_{3}^{\prime}$ in one point each.

The 3 lines determined by $\left|M_{t}, M_{r}^{\prime}\right|$ meet both $\Sigma_{3}$ and $\Sigma_{3}^{\prime}$ in two points each, and are the common chord-lines referred to above. 
The 9 lines given by

and

$$
{ }_{1} L_{1},{ }_{1} L_{2},{ }_{1} L_{3} ;{ }_{1} L_{1}^{\prime},{ }_{1} L_{2}^{\prime},{ }_{1} L_{3}^{\prime},
$$

form a trihedral pair.

(v) The homographic transformation : $\searrow_{3}$ and $\searrow_{3}^{\prime}$ intersect in one point.

Here there are no $F$-lines.

There are 6 chord-lines of $\searrow_{3}$ which are also chord-lines of $\Sigma_{j}$. Of the remaining 21 lines 15 must meet both $\Sigma_{3}$ and $\Sigma_{3}^{\prime}$ in one point, while 6 do not meet either.

\section{The group property.}

Any two of the above transformations successively performed lead to a transformation of the same set. The set of 5 therefore constitutes a group which is intimately associated with the 27 lines of the surface.

By taking a plane section of the surface the group of transformations is converted into a group of plane Cremona transformations of orders $4,8,12,16,20$. If $\Sigma_{3}$ and $\Sigma_{3}$ ' meet the plane in the points $A B C, A^{\prime} B^{\prime} C^{\prime}$, by taking these points as the $F$-points of a quadratic transformation we obtain a group of transformations of orders $2,4,6,8,10$.

If $C_{3}$ be the non-singular cubic in which $F_{3}$ intersects the plane, then it is easy to see that this cubic must be an invariant curve for all transformations of the group. The various $F$-points, in number 27 , all lie on the invariant cubic. 